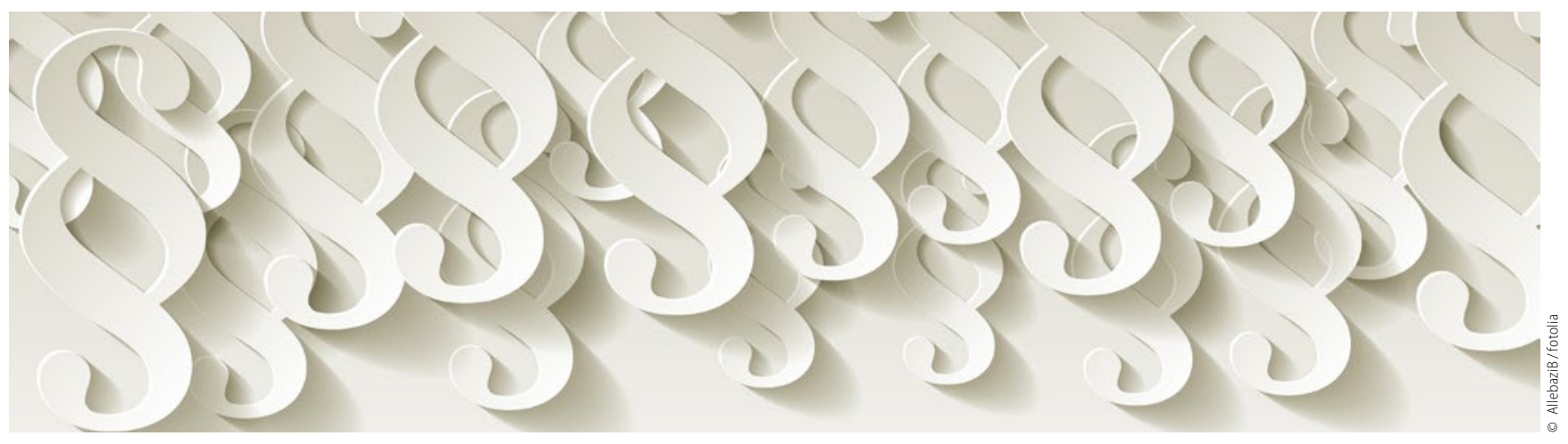

Beschäftigung von zwei angestellten Zahnärzten

\title{
Kein Vorbereitungsassistent möglich?
}

\begin{abstract}
In seinem Urteil vom 15. Januar 2016 (S 20 KA 5004/14) hat sich das Sozialgericht München mit der Frage befasst, ob ein Zahnarzt neben zwei angestellten Zahnärzten auch noch einen Vorbereitungsassistenten beschäftigen kann.
\end{abstract}

\section{Der Fall}

In dem konkreten Fall wurde einem Zahnarzt eine Vorbereitungsassistentin in Vollzeit genehmigt. In dem vom Zahnarzt gestellten Antrag wurde er darauf hingewiesen, dass die Genehmigung erlischt, wenn dadurch die Obergrenze von zwei vollzeitangestellten Zahnärzten überschritten wird. Dem Zahnarzt wurden darüber hinaus zwei angestellte Zahnärztinnen in Vollzeit genehmigt.

Mit Bescheid vom 23. Oktober 2013 wurde die Genehmigung der Vorbereitungsassistentin widerrufen, wobei zur Begründung auf die in Bayern maßgebliche Assistentenrichtlinie verwiesen wurde, nach der neben zwei ganztags angestellten Zahnärzten keine Genehmigung eines Vorbereitungsassistenten erfolgen könne.

Gegen den Bescheid erhob der Zahnarzt erfolglos Widerspruch, worauf er Klage beim zuständigen Sozialgericht München erhob.

\section{Die Entscheidung}

Nach Auffassung des Sozialgerichtes München war der Widerruf der Genehmigung einer Vorbereitungsassistentin nicht zu beanstanden.

In den tatsächlichen Verhältnissen, die der Genehmigung der Vorbereitungsassistentin zugrunde gelegen hätten, sei durch die Einstellung der zweiten angestellten Zahnärztin eine wesentliche Änderung eingetreten. Dies sei zu Recht zum Anlass genommen worden, die Genehmigung der Vorbereitungsassistentin mit Wirkung für die Zukunft durch Widerruf aufzuheben. Der Zahnarzt hätte, nachdem er die zweite Zahnärztin in Vollzeit angestellt habe, keinen Anspruch mehr auf die Genehmigung der Vorbereitungsassistentin gehabt.

Rechtsgrundlage für den vom Zahnarzt geltend gemachten Anspruch auf Genehmigung der Vorbereitungsassistentin sei $\$ 2$ Absatz 2 Satz 1 Zahnärzte-Zulassungsverordnung (ZV). Gemäß $₫ 32$ b Absatz 1 Satz 2 Zahnärzte-ZV in Verbindung mit $₫ 4$
Absatz 1 Satz 7 Bundesmantelvertrag für Zahnärzte (BMV-Z) könne ein Zahnarzt maximal zwei vollzeitbeschäftigte Zahnärzte anstellen.

Aus der Regelung zur Genehmigung von Vorbereitungsassistenten in $\$ 32$ Absatz 2 Satz 1 Zahnärzte-ZV und der zur Anstellung von Zahnärzten in $\$ 32 \mathrm{~b}$ Absatz $1 \mathrm{ZÄ}-Z \mathrm{ZV}$ ergebe sich nicht direkt, inwieweit die zahlenmäßige Beschränkung auf zwei vollzeitbeschäftigte angestellte Zahnärzte in $\$ 4 \mathrm{BMV}-\mathrm{Z}$ auch für die Genehmigung von Vorbereitungsassistenten gelte. Dies müsse nach dem Sinn und Zweck des Genehmigungserfordernisses unter Berücksichtigung der damit im Gesamtzusammenhang stehenden Bestimmungen beurteilt werden. Auch die Tätigkeit eines Vorbereitungsassistenten führe zu einer in gewissen Grenzen selbstständigen Behandlung der Versicherten. Dementsprechend würden diese Leistungen über den beschäftigenden Vertragszahnarzt als innerhalb des Versorgungssystems erbrachte Behandlungen vergütet. Im Lichte der Regelungen der $\$ \$ 32$, 32b, $3 \mathrm{ZÄ}-\mathrm{ZV}$ sei davon auszugehen, dass die zahlenmäßige Beschränkung des $\$ 4$ Absatz 1 Satz 7 BMV-Z auch für die Genehmigung eines Vorbereitungsassistenten gelte. Auf die Rechtmäßigkeit der Assistentenrichtlinie komme es nicht an, so dass der vom Zahnarzt gerügte Verstoß der Richtlinie gegen die Berufsausübungsfreiheit und den Gleichheitssatz dahinstehen könne.

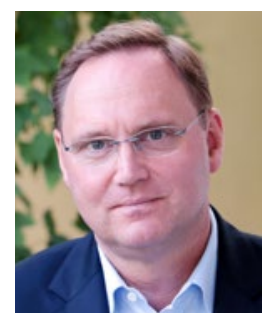

RA Michael Lennartz

lennmed.de Rechtsanwälte 